\title{
Effects of filler type in naming: Change in time criterion or attentional control of pathways?
}

\author{
SACHIKO KINOSHITA \\ Macquarie University, Sydney, New South Wales, Australia \\ and \\ STEPHEN J. LUPKER \\ University of Western Ontario, London, Ontario, Canada
}

\begin{abstract}
We report two naming experiments examining the effects of filler type on the size of regularity and frequency effects. Low-frequency exception words were used as one filler type in both experiments. Their effects were contrasted with the effects of nonword fillers (Experiment 1) and low-frequency regular word fillers (Experiment 2). In both experiments, the size of the regularity effect was unaffected by the filler type manipulation. In contrast, the frequency effect interacted with filler type such that relative to the low-frequency exception filler environment, the size of the frequency effect was reduced in the environment of low frequency regular word fillers, but not in the environment of nonword fillers. These results appear to be better explained in terms of Lupker, Brown, and Colombo's (1997) flexible timecriterion framework than in terms of a pathway control hypothesis (e.g., Zevin \& Balota, 2000).
\end{abstract}

A recurring theme in the word recognition literature is the proposal that readers can strategically shift their reliance on whole-word specific information ("lexical" information) versus sublexical information in reading aloud (Baluch \& Besner, 1991; Monsell, Patterson, Graham, Hughes, \& Milroy, 1992; Rastle \& Coltheart, 1999; Tabossi \& Laghi, 1992; Zevin \& Balota, 2000). Following Zevin and Balota, we will refer to this proposal as the attentional control of pathways hypothesis.

Traditionally, this notion has been examined within the dual-route framework (Coltheart, 1978; Coltheart, Curtis, Atkins, \& Haller, 1993; Coltheart, Rastle, Perry, Langdon, $\&$ Ziegler, 2001), which assumes that readers have at their disposal two ways of generating the pronunciation of a printed letter string. One way is referred to as the lexical route, in which the reader retrieves the whole-word phonology that is stored in the phonological output lexicon via the link from the orthographic input lexicon. The second way is referred to as the nonlexical route, in which the pronunciation is computed by means of an application of

S.K. and S.J.L. contributed equally to the writing of the paper; the order of authorship was alphabetical. We would like to thank Debra Jared, Michael Masson, and an anonymous reviewer for their helpful comments on an earlier version of the paper. Thanks are also due to Tamsen Taylor, Carl Windhorst, Anna Woollams, and Judy Wilson for research assistance. Correspondence should be addressed either to S. Kinoshita, Macquarie Centre for Cognitive Science (MACCS) and Department of Psychology, Macquarie University, Sydney 2109, NSW, Australia, or S. J. Lupker, University of Western Ontario, London, ON, N6A 5C2, Canada (e-mail: sachiko.kinoshita@mq.edu.au or lupker@uwo.ca). grapheme-phoneme correspondencerules. Exception words whose pronunciations do not follow the regular graphemephoneme correspondence rules (e.g., yacht, pint) can be read aloud correctly only via the lexical route. Nonwords (e.g., slint), which are not represented in the orthographic input lexicon, can only be read aloud correctly via the nonlexical route.

As Zevin and Balota (2000) have pointed out, it is also possible to examine the attentional control of pathways hypothesis within the framework of parallel-distributed processing (PDP) models. A major difference between these types of models and dual-route models, however, is that PDP models do not separate the actions of the two routes as fully as dual-route models do. Hence, the predictions made by the attentional control hypothesis within a PDP framework are less clear. Nonetheless, the present data will allow an evaluation of this hypothesis within a PDP framework. That evaluation will be presented in the General Discussion.

One method that has been used in studies that investigated the attentional control of pathways in normal readers is to compare performance in blocks composed entirely of one type of stimulus (pure blocks) with performance in blocks containing various types of stimuli (mixed blocks). These studies (e.g., Monsell et al., 1992; Rastle \& Coltheart, 1999) have demonstrated that performance for at least certain types of stimuli is better when those stimuli are named in pure blocks. These results have been interpreted as indicating that readers can strategically "turn down" reliance on the nonlexical route when they know in advance that it is advantageous to do so (as in a pure block of exception words). That is, the standard assumption here 
is that the lexical route is more automatized than the nonlexical route and, hence, only the nonlexical route is under strategic control. For purposes of the present discussion, we will adopt the same assumption.

More recently, however, Lupker and colleagues (Lupker, Brown, \& Colombo, 1997; Taylor \& Lupker, 2001) have suggested that these blocking effects are better interpreted within an alternative framework. According to their flexible time-criterion framework, readers do not always initiate articulation as soon as they are ready. Instead, there is a time criterion that guides the initiation of articulation. What readers do is to attempt to start articulation when that time criterion is reached. The position of the time criterion is based on the relative difficulty of the stimuli in the trial block: That is, it will correspond to a reaction time that is most appropriate for the particular stimuli in the trial block. The position of the time criterion is adjusted on a trial-by-trial basis such that following the naming of a rapidly named stimulus, the time criterion would be set lower than it would be following the naming of a more slowly named stimulus. Consequently, in a pure block of fast stimuli (e.g., words), the naming latency would be faster than if the same stimuli had been mixed with slower stimuli (e.g., nonwords). Conversely, a pure block of slow stimuli would produce naming latencies slower than had the same stimuli been mixed with faster stimuli. We refer to these types of results as "blocking effects."

In general, most blocking effects can be interpreted either in terms of attentional control of pathways or in terms of the flexible time-criterion framework. Nonetheless, Lupker et al. (1997) have been able to directly contrast the two hypotheses. For example, in their Experiment 3, highand low-frequency exception words were presented in either pure blocks or mixed blocks. According to the attentional control hypothesis, because both stimulus types are exception words, reliance on the nonlexical route would be minimal in all blocks. Thus, there should be no blocking effects. In contrast, the time-criterion hypothesis would predict blocking effects. In particular, the time criterion would be set at a lower position in the pure block of highfrequency words than in the mixed block, meaning that high-frequency words should be named faster in pure blocks. In contrast, the time criterion would be set at a higher position in the pure block of low-frequency words than in the mixed block, meaning that the low-frequency words should be named faster in mixed blocks. The results were fully consistent with the time-criterion account.

It should be noted, however, that even if the time-criterion account provides a better explanation of blocking effects, a blocking manipulation is not the only way to determine whether readers really can control their pathways to pronunciation. Another approach that has been used is to see whether the size of effects that are assumed to reflect the relative contributions of the lexical and the nonlexical pathways can be modulated by changing the nature of the filler stimuli within the block. In principle, the time-criterion framework would predict main effects of filler type (i.e., overall latencies for target stimuli should vary as a func- tion of the speed with which the fillers are named), but it would not necessarily predict any modulation in the size of effects for the targets as a function of filler type. The issue of the modulation of effect sizes is the focus of the present investigation.

To date, previous studies have mainly focused on the modulation of two such effects - namely, the regularity effect and the frequency effect. The regularity effect refers to the finding that words that follow regular graphemephoneme correspondence rules (e.g., pink) are named faster than words that do not (e.g., pint). This finding is interpreted within the dual-route framework as being due to a conflict in the pronunciation generated by the lexical route and the nonlexical route in the naming exception words. The fact that the regularity effect is generally observed only for low-frequency words (Seidenberg, Waters, Barnes, \& Tanenhaus, 1984; but see Jared, 1997, 2002) is explained within the dual-route framework by assuming that for high-frequency words the retrieval of pronunciation via the lexical route occurs well before the incompatible pronunciation computed by the nonlexical route creates a conflict. If this analysis is correct, the implication is that for low-frequency words, a manipulation that slows down the nonlexical route should reduce the size of the regularity effect (and conversely, a manipulation that speeds up the nonlexical route should magnify the size of the regularity effect).

Previous studies that examined the modulation of the regularity effect as a function of the composition of stimuli in a block have generally yielded little evidence of such modulation. ${ }^{1}$ For example, Coltheart and Rastle (1994) embedded low-frequency regular and exception target words in a block of high-frequency exception word fillers and a block of nonword fillers and found no interaction between filler type and regularity. Woollams and Kinoshita (1997) used the Coltheart and Rastle (1994) target stimuli and embedded them in a block of low-frequency exception word fillers and a block of nonword fillers and found no modulation of the size of the regularity effect. Jared (1997) used consistency of body-rime mapping rather than regularity of grapheme-phoneme correspondence and reported that the size of the consistency effect was not modulated by whether the fillers were nonwords or low-frequency exception words.

In contrast to these failures to strategically alter the size of the regularity effect, there have been demonstrations of a modulation of the size of the frequency effect as a function of filler type (Baluch \& Besner, 1991; Decker, Simpson, Yates, \& Adamopolous, 1999; Simpson \& Kang, 1994). According to the dual-route framework, frequency effects are lexical route effects. That is, high-frequency words are named more rapidly than low-frequency words due to the fact that high-frequency words are processed more rapidly than low-frequency words by the lexical route. Nonetheless, according to the dual-route framework, the size of the frequency effect for words that follow standard graphemephoneme rules (i.e., regular words) can be modulated if the contributions of the nonlexical route are altered. That 
is, processing on the nonlexical route can support processing on the lexical route, facilitating naming of lowfrequency regular words and, hence, decreasing the size of the frequency effect. The implication is that any manipulation that speeds up the nonlexical route should reduce the size of the regular word frequency effect (and conversely, any manipulation that slows down the nonlexical route should magnify the size of the frequency effect).

The results reported by Baluch and Besner (1991), Decker et al. (1999), and Simpson and Kang (1994) are all consistent with this prediction. Specifically, in these experiments, participants named target words having regular grapheme-phoneme mappings in one of two filler conditions. In one, the fillers were words with opaque graphemephoneme mappings and, hence, naming them would require the use of the lexical route. In the second, the fillers were either nonwords or other words with regular grapheme-phoneme mappings, both of which can be named via the nonlexical route. A reduced frequency effect for the target words was observed in the second condition. Since nonword and regular word fillers should promote a greater relative emphasis of the nonlexical route, these results are as predicted by the attentional control hypothesis.

In summary, there is a discrepancy between the effects of a filler type manipulation on the modulation of the regularity effect on the one hand and the frequency effect (for regular words) on the other. The studies that focused on the regularity effect would lead one to conclude that strategic control of pathways is not possible, whereas the studies that focused on the frequency effect would lead one to conclude otherwise. One question that naturally comes to mind is whether the frequency effect is simply easier to modulate than the regularity effect. Because all of the research described here (with the exception of the study by Jared, 1997 , to be discussed shortly) was aimed at demonstrating the modulation of a single effect, the answer to this question is unknown.

In contrast to the studies mentioned above, Jared (1997) did examine consistency and frequency effects simultaneously using a filler type manipulation. Her results provide support for the idea that the frequency effect is more sensitive than the consistency effect to a manipulation of filler type. (Note that it is only her Experiment 2 that allows this question to be evaluated, because her other experiments did not present high- and low-frequency words in the same block.)

In one sense, then, the present experiments, in which the sensitivity of regularity and frequency effects to a filler type manipulation were simultaneously examined, parallel and extend Jared's (1997) results. However, there are at least two key differences between her experiment and the present experiments. First, because the aim of Jared's (1997) study was not specifically to test the time-criterion account, there was no attempt to control the speed of naming of the different filler types (high- and low-frequency inconsistent words and nonwords). It is therefore unclear whether the effect of filler type she observed (a reduced frequency effect with nonword fillers) was an effect of filler speed, as suggested by the time-criterion account, or an effect of filler type, as suggested by the pathway control hypothesis.

Second, Jared (1997) manipulated consistency of body-rime mapping, rather than regularity of graphemephoneme correspondences. This was because the theoretical framework she was using to test the pathway control hypothesis was the PDP framework, and within this framework, the consistency of body-rime units is a more central factor than regularity. Although consistency and regularity are highly correlated, the two variables are different, as indicated by the fact that some of her inconsistent target words (e.g., raid, crave) were regular. From the dual-route perspective (e.g., Coltheart et al., 1993), the regularity of grapheme-phoneme correspondences, rather than the consistency of body-rime units, is the critical variable that distinguishes the lexical and nonlexical routes (although see Coltheart et al., 2001). Thus, to test the pathway control hypothesis within the standard dualroute framework, it is important to examine whether there is a modulation of the regularity effect, rather than the consistency effect.

The initial aim of the present research was therefore empirical: We wished to examine whether both the size of the frequency effect for regular words and the size of the regularity effect for low-frequency words can be modulated simultaneously under identical conditions. To this end, we used three types of target words: high-frequency regular, low-frequency regular, and low-frequency exception words. The comparison between the first two types of words provided a measure of the size of the regular word frequency effect, and the comparison between the latter two provided a measure of the low-frequency regularity effect. These critical target words were embedded in one of two types of fillers. In Experiment 1, we used low-frequency exception word fillers and nonword fillers because these fillers should provide the maximum motivation for adjusting the attention allocated to the nonlexical route.

On the basis of the majority of the results reported above, we would not necessarily expect a modulation in the size of regularity effect as a function of filler type, but would expect the size of frequency effect to be reduced in the block with nonword fillers. In contrast, if the attentional control hypothesis is correct we would expect both effects to be modulated and for the effect sizes to go in opposite directions. With respect to the size of the frequency effect, with nonword fillers, the nonlexical route would presumably receive additional attention, which should aid naming of the low-frequency words much more than the naming of high-frequency words. Thus, the size of the frequency effect should decrease. With respect to the size of the regularity effect, when the nonlexical route receives greater attention due to the use of nonword fillers, the exception words should pay an increased penalty due to increased competition. As a result, latencies for the lowfrequency exception words should increase, producing an increased regularity effect. 
Table 1

Stimulus Characteristics of Targets and Fillers Used

\begin{tabular}{lrrrr}
\hline \multicolumn{1}{c}{ Variable } & Length & \multicolumn{1}{c}{ Freq } & $N$ & Bigram \\
\hline Word Target & & & & \\
$\quad$ High-frequency regular & 4.57 & 2509.33 & 5.27 & 682.97 \\
Low-frequency regular & 4.60 & 117.70 & 5.03 & 613.23 \\
$\quad$ Low-frequency exception & 4.60 & 113.97 & 5.00 & 623.40 \\
Fillers & & & & \\
$\quad$ Low-frequency exception & 4.80 & 106.18 & 3.17 & 661.24 \\
$\quad$ Nonword & 4.80 & - & 6.33 & 736.58 \\
$\quad$ Low frequency regular & 4.78 & 110.86 & 6.07 & 775.00 \\
\hline
\end{tabular}

Note-Length, item length (number of letters); Freq, Celex written frequency (per 18 million); $N$, number of orthographic neighbors; Bigram, bigram frequency.

Finally, with respect to the time-criterion account, we would predict no effects of filler type at all. This prediction is based on the fact that our filler words and nonwords were selected to have approximately the same overall latencies based on their means in previous experiments. Thus, the time criterion should be in virtually the same place in both filler conditions.

\section{EXPERIMENT 1}

\section{Method}

Design. The present experiment featured a 3 (target type: highfrequency regular words, low-frequency regular words, lowfrequency exception words) $\times 2$ (filler type: exception words vs. nonwords) factorial design, with both factors being within-subjects factors. The dependent variables were naming latency and error rate.

Participants. Twenty-four psychology students from Macquarie University participated in the experiment for course credit. All participants were native Australian-English speakers.

Materials. The targets were 30 high-frequency regular, 30 lowfrequency regular, and 30 low-frequency exception words. All words were monosyllabic, and were four to six letters long. The three types of words were matched on initial phoneme, number of letters, bigram frequency, and $N$ (the number of orthographic neighbors of the same length). The low-frequency regular and exception words were also matched on frequency based on the Celex database (Baayen, Piepenbrock, \& van Rijn, 1993). The stimulus characteristics are shown in Table 1, and the items are listed in the Appendix A. In addition to the targets, there were 45 exception word fillers and 45 nonword fillers. The two types of fillers were matched on length and initial phoneme. Their characteristics are also shown in Table 1, and the items are listed in Appendix B.

The targets were divided into two equal-size sets, Sets A and B, each containing 15 high-frequency regular, 15 low-frequency regular, and 15 low-frequency exception words. The assignment of the sets to the two filler type blocks was counterbalanced across participants so that for half of the participants, Set A items appeared with the exception word fillers and Set B items appeared with the nonword fillers; for the other half, the assignment was reversed.

There were also 12 practice stimuli in each filler type block that preceded the test stimuli. The practice stimuli included the same type of fillers as the test block (i.e., either low-frequency exception words or nonwords) and one each of high-frequency regular, lowfrequency regular, and low-frequency exception words. None of the practice stimuli were the same as the experimental stimuli.

Apparatus and Procedure. Each participant completed two blocks of trials, one containing the exception word fillers and the other containing the nonword fillers. Each block consisted of 12 practice trials and 90 test trials.
Half of the participants received the exception filler block first, and the other half received the nonword filler block first. With the counterbalancing of assignment of sets of critical targets to the two filler type blocks, this resulted in four different subgroups of participants for counterbalancing purposes.

At the outset of the experiment, participants were told that a list of words and nonwords would be shown on the computer screen, one at a time. Participants were instructed to read aloud each item as soon as possible without making too many errors.

Participants were tested individually. Instructions and stimuli were presented on an NEC 4FG Multisync monitor, and reaction time data were recorded to the nearest millisecond using the DMASTR display system developed by K.I. Forster and J.C. Forster at Monash University, Australia, and the University of Arizona (details of this system can be obtained at the following Internet address: http:// www.u.arizona.edu/ kforster/dmastr/dmastr.htm) running on a Deltacom 486 IBM-compatible computer. Reaction times were recorded using an amplitude voice key fitted to each participant and held a constant distance from the mouth throughout the experiment by means of a headset. Naming errors and possible measurement errors due to inappropriate voice key activation (e.g., coughing) were recorded manually by the experimenter. Each trial started with the presentation of a fixation signal (+) for $500 \mathrm{msec}$, followed by a target. The target remained on the screen for a maximum of 2,000 msec, or until the voice key was triggered by participant's response. Participants were given no feedback on either naming latencies or error rates during the experiment.

\section{Results}

For this and subsequent analyses, the preliminary treatment of trials was as follows. Any trial on which a participant or voice key error occurred was excluded from the latency analysis. Voice key errors occurred on $0.37 \%$ of the trials in the exception word filler condition and $0.09 \%$ of the trials on the nonword filler condition. In order to reduce effects of outliers, spuriously long or short reaction times were trimmed to the cutoff value of $2 S D$ above or below the mean for each participant. This procedure affected $3.3 \%$ of trials in the exception word filler condition and $4.17 \%$ of trials in the nonword filler condition. Both analyses treating subjects as a random variable $\left(F_{\mathrm{s}}\right)$ and those treating items as a random variable $\left(F_{\mathrm{i}}\right)$ are reported. For each of naming latency and percent error rate, we report first a 3 (target type) $\times 2$ (filler type) analysis of variance (ANOVA), then two $2 \times 2$ ANOVAs, one including only low-frequency words and another including only regular words, to examine the modulation of the regularity effect and the modulation of the frequency effect. In the subject analysis $\left(F_{\mathrm{s}}\right)$, both the target type and filler type factors were treated as within-subjects factors; in the item analysis $\left(F_{\mathrm{i}}\right)$, target type was treated as a between-items factor and filler type as a within-items factor. An alpha level of .05 was used. The mean naming latencies and mean percent error rates from the subject analysis are presented in Table 2.

Targets. For naming latency, the 3 (target type) $\times 2$ (filler type) ANOVA showed that the main effect of target type was significant $\left[F_{\mathrm{s}}(2,46)=45.26, M S_{\mathrm{e}}=333.07\right.$; $\left.F_{\mathrm{i}}(2,87)=9.79, M S_{\mathrm{e}}=2379.36\right]$. The main effect of filler type was nonsignificant $\left[F_{\mathrm{s}}(1,23)<1.0 ; F_{\mathrm{i}}(1,87)<1.0\right]$. The interaction between these two factors was nonsignificant $\left[F_{\mathrm{s}}(2,46)<1.0, F_{\mathrm{i}}(2,87)=1.16, M S_{\mathrm{e}}=492.40\right]$. 
Table 2

Mean Naming Reaction Times (RT, in Milliseconds) and Percent Errors (\%E) in Experiment 1

\begin{tabular}{|c|c|c|c|c|c|c|}
\hline \multirow[b]{3}{*}{ Target Type } & \multicolumn{4}{|c|}{ Filler Type } & & \\
\hline & \multicolumn{2}{|c|}{ EXC } & \multicolumn{2}{|c|}{ NW } & \multicolumn{2}{|c|}{ Difference } \\
\hline & RT & $\% \mathrm{E}$ & RT & $\% \mathrm{E}$ & RT & $\% \mathrm{E}$ \\
\hline High-frequency regular & 477 & 1.1 & 483 & 1.4 & -6 & -.3 \\
\hline Low-frequency regular & 487 & 2.0 & 494 & 2.0 & -7 & .0 \\
\hline Low-frequency exception & 515 & 22.2 & 514 & 27.0 & 1 & -4.8 \\
\hline Frequency effect & 10 & .9 & 11 & .6 & & \\
\hline Regularity effect & 28 & 20.2 & 20 & 25.0 & & \\
\hline Fillers & 510 & 17.5 & 503 & 6.0 & 7 & 11.5 \\
\hline
\end{tabular}

Note-EXC, low-frequency exception words; NW, nonwords.

The 2 (regularity) $\times 2$ (filler type) ANOVA including only low-frequency words revealed a significant main effect of regularity $\left[F_{\mathrm{s}}(1,23)=37.11, M S_{\mathrm{e}}=364.13 ; F_{\mathrm{i}}(1,58)=\right.$ $\left.7.69, M S_{\mathrm{e}}=2,906.04\right]$. The interaction between regularity and filler type was nonsignificant $\left[F_{\mathrm{s}}(1,23)<1.0\right.$; $\left.F_{\mathrm{i}}(1,58)=1.70, M S_{\mathrm{e}}=567.38\right]$.

The 2 (frequency) $\times 2$ (filler type) ANOVA including only regular words revealed a significant effect of frequency by subjects $\left[F_{\mathrm{s}}(1,23)=12.29, M S_{\mathrm{e}}=233.59\right]$ and a marginal effect by items $\left[F_{\mathrm{i}}(1,58)=3.29, M S_{\mathrm{e}}=275.78\right.$, $p=.07]$. The interaction between frequency and filler type was nonsignificant $\left[F_{\mathrm{s}}(1,23)<1.0 ; F_{\mathrm{i}}(1,58)<1.0\right]$.

For error rate, the 3 (target type) $\times 2$ (filler type) ANOVA showed that the main effect of target type was significant $\left[F_{\mathrm{s}}(2,46)=70.41, M S_{\mathrm{e}}=120.12 ; F_{\mathrm{i}}(2,87)=\right.$ $\left.30.37, M S_{\mathrm{e}}=41.78\right]$. The main effect of filler type was nonsignificant $\left[F_{\mathrm{s}}(1,23)=2.01, M S_{\mathrm{e}}=49.78 ; F_{\mathrm{i}}(1,87)=\right.$ $\left.2.23, M S_{\mathrm{e}}=6.73\right]$. The interaction between these two factors was nonsignificant $\left[F_{\mathrm{s}}(2,46)=1.53, M S_{\mathrm{e}}=55.29\right.$; $\left.F_{\mathrm{i}}(2,87)=1.52, M S_{\mathrm{e}}=6.73\right]$.

The 2 (regularity) $\times 2$ (filler type) ANOVA revealed a significant effect of regularity $\left[F_{\mathrm{s}}(1,23)=76.64, M S_{\mathrm{e}}=\right.$ $\left.160.44 ; F_{\mathrm{i}}(1,58)=29.65, M S_{\mathrm{e}}=62.23\right]$. The interaction between regularity and filler type was nonsignificant $\left[F_{\mathrm{s}}(1,23)=2.19, M S_{\mathrm{e}}=61.29 ; F_{\mathrm{i}}(1,58)=2.13, M S_{\mathrm{e}}=9.43\right]$.

The 2 (frequency) $\times 2$ (filler type) ANOVA showed no significant effect of frequency $\left[F_{\mathrm{s}}(1,23)=1.09, M S_{\mathrm{e}}=\right.$ $\left.10.64 ; F_{\mathrm{i}}(1,58)<1.0\right]$. The interaction between frequency and filler type was nonsignificant $\left[F_{\mathrm{s}}(1,23)<1.0\right.$; $\left.F_{\mathrm{i}}(1,58)<1.0\right]$.

Fillers. For naming latency, the effect of filler type was nonsignificant $\left[F_{\mathrm{s}}(1,23)=1.43, M S_{\mathrm{e}}=376.98 ; F_{\mathrm{i}}(1,88)=\right.$ $\left.1.46, M S_{\mathrm{e}}=2088.02\right]$. For errors, the effect was significant $\left[F_{\mathrm{s}}(1,23)=31.89, M S_{\mathrm{e}}=49.69 ; F_{\mathrm{i}}(1,88)=12.06\right.$, $\left.M S_{\mathrm{e}}=245.88\right]$. There were $12 \%$ more errors to the exception word fillers than to the nonword fillers.

\section{Discussion}

Three points should be made about the results from Experiment 1 . First, as hoped, the target stimuli produced a clear regularity effect for the low-frequency words and a clear frequency effect for the regular words. Second, as hoped, the latencies for the exception word fillers $(510 \mathrm{msec})$ and the nonword fillers (503 msec) were fairly similar. Finally, and most importantly, there was little evidence that either the regularity effect or the frequency effect was modulated, as a function of filler type, in the way predicted by the attentional control hypothesis. In fact, to the extent that there was a modulation at all, the effects went in the direction opposite to the predictions made by that account. That is, the regularity effect decreased by $8 \mathrm{msec}$ and the frequency effect increased by $1 \mathrm{msec}$ when nonword fillers were used.

The fact that the inclusion of nonword fillers did not increase the size of the regularity effect is, perhaps, not surprising given previous results (e.g., Coltheart \& Rastle, 1994). What is a bit more surprising was our failure to modulate the size of the frequency effect. As noted, Baluch and Besner (1991) in Persian, Simpson and Kang (1994) in Korean and, more recently, Decker et al. (1999) in English have all reported the modulation of frequency effects. A further examination of the details of those experiments appears to be in order.

In all of these experiments, the authors demonstrated a reduction in the frequency effect for words that can, in theory, be named by the nonlexical route when fillers that should bias participants toward use of that route are used. Baluch and Besner (1991) demonstrated a reduction in the frequency effect for "transparent" Persian words (i.e., words containing the graphemes for all their phonemes) when nonwords were used as fillers (in contrast to a nofiller condition). Simpson and Kang (1994) demonstrated a reduction in the frequency effect for Hangul words when those words were mixed with either other Hangul words or nonwords (in contrast to when the fillers were written in Hanza, a logographic script). Finally Decker et al. (1999) demonstrated a reduction in the frequency effect for regular words in English when those words were mixed with regular words (in contrast to using exception word fillers). Thus, the reduced frequency effect pattern does appear to manifest across a number of situations.

What is also true about these situations, however, with one possible exception (the comparison between the Hangul word and nonword filler conditions in the Simpson \& Kang, 1994, study, to be discussed in the General Discussion), is that the overall latencies in the filler condition that showed the reduced frequency effect were substantially shorter than those for the other filler condition. The question that this raises, of course, is why the frequency effect would be reduced in the faster filler condition. A possible answer is that it might be a time-criterion effect. That is, due to the fact that the fillers in the condition with the reduced frequency effect had shorter latencies than the fillers in the other condition, the time criterion would have been set at a lower point in the former condition than in the latter condition. As a result, both low- and high-frequency words produced shorter latencies. However, the speedup for low-frequency words was larger than that for highfrequency words. This could be because the high-frequency 
words are faster than all other item types (including the fast fillers) and, hence, there is less scope for speedup for these words.

\section{EXPERIMENT 2}

If our analysis of the impact of the time criterion in the previous literature is correct, it would explain why we did not observe a reduction in the frequency effect in Experiment 1. As noted, our nonwords and exception words were selected so as to have essentially equivalent latencies. The reason was that we didn't want our filler manipulation to be contaminated by participants using different placements of their time criteria in the two conditions. Results suggest that we were fairly successful. Thus, in contrast to virtually all the experiments showing a reduced frequency effect, we did not create conditions that would cause participants to decrease their overall naming latencies in the condition that was supposed to show a reduced frequency effect.

This analysis also suggests a way to produce a reduced frequency effect for our regular word targets. Rather than using nonword fillers, as in Baluch and Besner's (1991) experiment, we may be able to create the reduced frequency effect if we use regular word fillers, as in Simpson and Kang's (1994) experiment and Decker et al.'s (1999) experiment. In English at least, regular words are named substantially faster than nonwords. Thus, we can expect that the time criterion will be set lower in the regular word filler condition than in the nonword filler condition (and hence, lower than in the exception word filler condition). If our analysis is correct, we should now observe a reduced frequency effect in the regular word filler condition.

Note that this is the same prediction that would be made by the attentional control hypothesis. According to dualroute logic, as employed by Simpson and Kang (1994) and by Decker et al. (1999), words that have transparent spelling-to-sound relationships should effectively bias participants more toward the use of the nonlexical route than exception words would. Thus, either regular word or nonword fillers would be expected to produce a smaller regular word frequency effect than exception word fillers.

Experiment 2 was an attempt to test this prediction using the identical stimuli as in Experiment 1. Note that if we do observe a reduced frequency effect in the regular word filler condition in Experiment 2, the inclusion of exception word targets will provide a way to distinguish between these two accounts of that result. That is, the inclusion of these words will allow us to determine whether we simultaneously observe an increased regularity effect. If the reason for the decreased frequency effect is more extensive use of the nonlexical route in the regular word filler condition, the low-frequency exception words should suffer increased competition from that route. Thus, one would expect the latency difference between the exception words and the regular words to increase. On the other hand, if the only reason for the reduced frequency effect in the regular word filler condition is that participants are setting a lower time criterion, there is no reason to believe that the exception word fillers won't also benefit from this lower time criterion. Thus, there would be no reason to expect the low-frequency regularity effect to change in the regular word filler condition.

\section{Method}

Design. The present experiment featured a 3 (target type: highfrequency regular, low-frequency regular, low-frequency exception) $\times 2$ (filler type: low-frequency exception words vs. low-frequency regular words) factorial design, with both factors being withinsubjects factors.

Participants. An additional 24 volunteer 1st-year psychology students from the same population as Experiment 1 participated in Experiment 2.

Materials. Except for the fact that the nonword fillers used in Experiment 1 were replaced by low-frequency regular word fillers, the materials were identical to those of Experiment 1. The regular word fillers were selected to match the exception word fillers on length, initial phoneme, and frequency. Their stimulus characteristics are shown in Table 1, and the items are listed in Appendix B.

Apparatus and Procedure. The apparatus and procedure were identical to those of Experiment 1.

\section{Results}

The preliminary treatment of data was as in Experiment 1. Voice key errors occurred on $0.33 \%$ of trials in both the exception word filler condition and the regular word filler condition. The trimming procedure (which replaced spuriously long or short latencies with the cutoff value of $2 S D$ above or below the mean for each participant)replaced $3.95 \%$ of the trials in the exception word filler condition and $3.15 \%$ of the trials in the regular word filler condition. The mean naming latencies and mean percent error rates from the subject analysis are presented in Table 3.

Targets. For naming latency, the 3 (target type) $\times 2$ (filler type) ANOVA showed that the main effect of target type was significant $\left[F_{\mathrm{s}}(2,46)=40.58, M S_{\mathrm{e}}=783.09\right.$; $\left.F_{\mathrm{i}}(2,87)=14.35, M S_{\mathrm{e}}=3,891.58\right]$. The main effect of filler type was also significant $\left[F_{\mathrm{s}}(1,23)=14.92, M S_{\mathrm{e}}=\right.$ $\left.2,255.05 ; F_{\mathrm{i}}(1,87)=41.92, M S_{\mathrm{e}}=702.32\right]$. The interaction between these two factors was significant by subjects $\left[F_{\mathrm{s}}(2,46)=4.23, M S_{\mathrm{e}}=581.09\right]$, although not by items $\left[F_{\mathrm{i}}(2,87)=1.37, M S_{\mathrm{e}}=702.32\right]$.

The 2 (regularity) $\times 2$ (filler type) ANOVA including only the low-frequency words showed a significant main

Table 3

Mean Naming Reaction Times (RT, in Milliseconds) and Percent Errors (\%E) in Experiment 2

\begin{tabular}{|c|c|c|c|c|c|c|}
\hline \multirow[b]{3}{*}{ Target Type } & \multicolumn{4}{|c|}{ Filler Type } & & \\
\hline & \multicolumn{2}{|c|}{ EXC } & \multicolumn{2}{|c|}{ REG } & \multicolumn{2}{|c|}{ Difference } \\
\hline & RT & $\% \mathrm{E}$ & RT & $\% \mathrm{E}$ & RT & $\% \mathrm{E}$ \\
\hline High-frequency regular & 516 & .3 & 500 & 1.7 & 16 & -1.4 \\
\hline Low-frequency regular & 540 & .3 & 510 & 2.0 & 30 & -1.7 \\
\hline Low-frequency exception & 582 & 16.7 & 552 & 19.5 & 30 & -2.8 \\
\hline Frequency-effect & 24 & 0 & 10 & 0.3 & & \\
\hline Regularity-effect & 42 & 16.4 & 42 & 17.5 & & \\
\hline Fillers & 587 & 13.6 & 519 & 2.7 & 68 & 10.9 \\
\hline
\end{tabular}

Note-EXC, low-frequency exception words; REG, low-frequency regular words. 
effect of regularity $\left[F_{\mathrm{s}}(1,23)=35.90, M S_{\mathrm{e}}=768.13\right.$; $\left.F_{\mathrm{i}}(1,58)=10.81, M S_{\mathrm{e}}=5,000.91\right]$. The interaction between regularity and filler type was nonsignificant $\left[F_{\mathrm{s}}(1,23)=\right.$ $\left.1.60, M S_{\mathrm{e}}=766.68 ; F_{\mathrm{i}}(1,58)<1.0\right]$.

The 2 (frequency) $\times 2$ (filler type) ANOVA including only the regular word targets showed a significant main effect of frequency $\left[F_{\mathrm{s}}(1,23)=19.48, M S_{\mathrm{e}}=338.86\right.$; $\left.F_{\mathrm{i}}(1,58)=4.94, M S_{\mathrm{e}}=1,700.54\right]$. The interaction between frequency and filler type was also significant $\left[F_{\mathrm{s}}(1,23)=\right.$ $\left.5.40, M S_{\mathrm{e}}=228.41 ; F_{\mathrm{i}}(1,58)=6.27, M S_{\mathrm{e}}=245.81\right] .^{2}$

For error rate, the 3 (target type) $\times 2$ (filler type) ANOVA showed that the main effect of target type was significant $\left[F_{\mathrm{s}}(2,46)=39.78, M S_{\mathrm{e}}=116.43 ; F_{\mathrm{i}}(2,87)=26.57, M S_{\mathrm{e}}=\right.$ 26.17]. The main effect of filler type did not reach significance by subjects $\left[F_{\mathrm{s}}(1,23)=3.07, M S_{\mathrm{e}}=44.65, p=.09\right]$, but was significant by items $\left[F_{\mathrm{i}}(1,87)=5.10, M S_{\mathrm{e}}=4.00\right]$. The interaction between these two factors was nonsignificant $\left[F_{\mathrm{s}}(2,46)<1.0 ; F_{\mathrm{i}}(2,87)<1.0\right]$.

The 2 (regularity) $\times 2$ (filler type) ANOVA including only the low-frequency word targets showed a significant main effect of regularity $\left[F_{\mathrm{s}}(1,23)=42.51, M S_{\mathrm{e}}=162.07\right.$; $\left.F_{\mathrm{i}}(1,58)=28.82, M S_{\mathrm{e}}=38.50\right]$. This effect was due to the greater error rate for the exception targets $(18.0 \%)$ than for the regular targets $(1.2 \%)$. The interaction between regularity and filler type was nonsignificant $\left[F_{\mathrm{s}}(1,23)<1.0\right.$; $\left.F_{\mathrm{i}}(1,58)<1.0\right]$.

The 2 (frequency) $\times 2$ (filler type) ANOVA including only the regular word targets showed no effect of frequency $\left[F_{\mathrm{s}}(1,23)<1.0 ; F_{\mathrm{i}}(1,58)<1.0\right]$. The interaction between frequency and filler type was also nonsignificant $\left[F_{\mathrm{s}}(1,23)<1.0 ; F_{\mathrm{i}}(1,58)<1.0\right]$.

Fillers. For naming latency, there was a significant effect of filler type $\left[F_{\mathrm{s}}(1,23)=19.23, M S_{\mathrm{e}}=2,874.23 ; F_{\mathrm{i}}(1,88)=\right.$ $\left.36.36, M S_{\mathrm{e}}=564.73\right]$. The exception word fillers were named $67 \mathrm{msec}$ more slowly than the regular word fillers. For errors, there was also a significant effect $\left[F_{\mathrm{s}}(1,23)=\right.$ $\left.46.88, M S_{\mathrm{e}}=30.60 ; F_{\mathrm{i}}(1,88)=16.66, M S_{\mathrm{e}}=38.67\right]$. There were $11 \%$ more errors to the exception word fillers than to the regular word fillers.

\section{Discussion}

The results of Experiment 2 showed that target words were named faster when the fillers were low-frequency regular words than when they were low-frequency exception words. Thus, our change of fillers from nonwords to low-frequency regular words did, indeed, create a situation similar to that created in prior investigations (i.e., Baluch \& Besner, 1991; Decker et al., 1999; Simpson \& Kang, 1994). More importantly, the size of the frequency effect (for low-frequency regular targets) was reduced in the regular word filler environment while the size of the regularity effect was unaffected by filler type (in fact, numerically, the regularity effect was absolutely identical in the two filler conditions - $42 \mathrm{msec})$. These results contrast with those of Experiment 1, in which neither the size of the frequency effect nor the size of the regularity effect was affected by the use of exception word fillers versus nonword fillers.
Our inability to once again modulate the size of the regularity effect while at the same time being able to modulate the size of the frequency effect is difficult to explain within the framework of the pathway control hypothesis. That is, whereas the results of Experiment 1 can be explained in terms of an ineffective manipulation, the results of Experiment 2 cannot be. If participants were really producing a decreased frequency effect through a more extensive use of their nonlexical pathway in the regular word filler condition, we should also have observed an increase in the size of the regularity effect. The fact that we did not makes it quite difficult to explain the reduction in the frequency effect in that fashion.

In fact, the results of Experiments 1 and 2, taken together, cause even more difficulty for the pathway control hypothesis. Specifically, it is unclear why the use of regular word fillers, but not nonword fillers, would produce a reduction in the frequency effect. According to the attentional control hypothesis, this reduction would be due to the increased use of the nonlexical route. If anything, however, it should be the use of nonword fillers, which can only be named via the nonlexical route, which should have provided the stronger encouragement for participants to use the nonlexical route rather than to use regular word fillers, which can be named via either the lexical or the nonlexical route.

In contrast, these results are quite consistent with our suggestion that a modulation of the frequency effect requires an overall reduction in latency and, hence, the results are amenable to a time-criterion explanation. That explanation would be based on the idea that the reduction in the size of the frequency effect is due to the fact that the highfrequency regular word targets have less potential to speed up than the more slowly named low-frequency regular word targets. That is, due to the fact that the regular word fillers are named much faster than the exception word fillers, there is a pull to speed up in the former condition. This pull affects all word targets, but the impact is smaller on the high-frequency regular word targets, which are faster to name than the (low-frequency) regular word fillers. This pattern is expected from the time-criterion account, which states that when fast and slow items are mixed, the time criterion is set at a point that is between the optimal criteria for the fast and slow items. As a result, the frequency effect, which is a contrast between high- and low-frequency words, will shrink, whereas the regularity effect, which is a contrast between two types of lowfrequency words, will not.

\section{GENERAL DISCUSSION}

Past research has indicated that, using a filler type manipulation, it is possible to modulate the size of the frequency effect in naming regular words (Baluch \& Besner, 1991; Decker et al., 1999; Simpson \& Kang, 1994) in a way that is consistent with the attentional control of pathways hypothesis. Research has also indicated that it is much more difficult to modulate the size of the regularity 
effect in the case of naming low-frequency words (e.g., Coltheart \& Rastle, 1994) using that same manipulation. The goal of the present paper was to examine this apparent paradox. The present results indicate that neither effect can be modulated by a filler type manipulation unless one type of filler is faster than the other. When the contrasting filler types differed in speed, although it was still not possible to modulate the size of the regularity effect, it was possible to modulate the size of the frequency effect. This modulation was due to the fact that latencies for the lowfrequency words sped up more than latencies for the highfrequency words.

These results suggest that the observed reduction in the frequency effect is essentially driven by the placement of the time criterion (Lupker et al., 1997). The position of the time criterion is a function of both the difficulty of the target stimuli and the difficulty of the fillers. When rapidly named fillers are used, the position of the time criterion will be lower and, hence, overall latencies will decrease. However, high-frequency regular words are already fast and if they are even faster than the faster fillers, they have less room for speedup. This is expected from the view that when items with varying latencies are mixed together, the time criterion will be set between the criteria that are optimal for the different item types. If the high-frequency target words are faster than the faster fillers, their latencies would not move as much as the slower low-frequency regular word targets, and as a result, the frequency effect is reduced.

A survey of the relevant literature indicates that this analysis may provide the resolution of the apparent paradox. Baluch and Besner (1991) contrasted nonword fillers with no fillers and found that participants were faster to name transparent Persian words and produced a smaller frequency effect with nonword fillers. The nonwords were actually named more rapidly than the low-frequency "transparent" Persian words in the no-filler condition. Thus, they would, presumably, have exerted a downward pull on at least those stimuli. However, an additional reason that the participants in the nonword filler condition were faster could have been practice effects. That is, because the nonword filler condition, by definition, required participants to name nonwords, there were twice as many trials (80) in this condition as in the no-filler condition (40). Thus, at least part of the reduction in overall latency (and in the size of the frequency effect) may have been caused by the participants becoming more practiced at the task.

Decker et al. (1999) contrasted regular and exception fillers and found that participants were much faster to name regular words and produced a smaller frequency effect when the fillers were regular words. In English, participants would have been able to name the regular fillers faster than the exception fillers. ${ }^{3}$ Thus, the time criterion would have been set much lower in the regular filler condition than in the exception filler condition, producing both faster latencies and a smaller frequency effect.

Simpson and Kang (1994) contrasted Hanza and Hangul fillers and found that participants were much faster to name Hangul targets and produced a smaller frequency ef- fect when the fillers were Hangul words. Since Hangul is a shallow orthography whereas Hanza is a logography, participants would have been able to name the Hangul fillers much faster than the Hanza fillers. Thus, the time criterion would have been lower in the Hangul filler condition, producing both faster latencies and a smaller frequency effect.

As mentioned earlier, the one potential exception to this characterization is Simpson and Kang's (1994) nonexistent frequency effect for Hangul words when these were mixed with nonwords. Overall latencies for these participants were slightly lower than for the participants in the Hanza filler condition (where the participants showed a 60-msec frequency effect) but were noticeably larger than for the participants in the Hangul filler condition (where there was a 21 -msec frequency effect). What complicates these contrasts, however, is the fact that the participant population in the nonword filler condition was quite different from that in the Hanza and Hangul filler conditions. Participants in the latter groups were students at a Korean University. Participants in the former group were Korean undergraduate or graduate students (or spouses) at an American University. These individuals were tested on their knowledge of Hanza and performed as well as those in the other two groups. However, they were not tested on their knowledge of Hangul, and hence there is no way of knowing whether they would have produced a frequency effect when naming Hangul words under any situation. Equally importantly, there is no way to meaningfully compare the overall latencies for these individuals, who no longer live in a Korean language environment, with the overall latencies of the other two groups.

We also note that the time-criterion account is consistent with the results reported recently by Zevin and Balota (2000, Experiment 3) using a different manipulation of list composition. Instead of mixing fillers and targets randomly, as was done in the aforementioned studies, they used a "priming" manipulation in which a sequence of five fillers of one type (nonwords or low-frequency exception words) preceded the presentation of a target. They reported a reduction in the frequency effect for target words (regular words), which they interpreted in terms of pathway control. We note, however, that their results are also consistent with the time-criterion hypothesis. Specifically, the results showed that their nonword primes were faster than the exception word primes, and that their lowfrequency regular word targets sped up (by $16 \mathrm{msec}$ ) in response to nonword primes more than the high-frequency regular word targets sped up (by $6 \mathrm{msec}$ ). Thus, again, the reduction in the frequency effect was observed in the faster filler condition.

Finally, we note that recently, Raman, Baluch, and Besner (2000) reported data that, they argued, were more compatible with the time-criterion account than the pathway control hypothesis. In their study, native Turkish readers read aloud high-and low-frequency Turkish words and Turkish nonwords. The critical result was that the frequency effect was not eliminated when the words were 
mixed with nonwords that were matched in latency to the word stimuli. Raman et al. argued that because Turkish is a completely transparent orthography, the pathway control hypothesis predicts a complete elimination of the frequency effect when words are mixed with nonwords. On the other hand, because the nonword stimuli were matched in latency to the word stimuli, the time-criterion hypothesis would predict the presence of the frequency effect, as was observed. In sum, it appears that all of the existing data concerning the interaction between filler type and frequency are compatible with the time-criterion account.

\section{The Attentional Control of Pathways Hypothesis}

The attentional control hypothesis is based on the idea that participants can alter the relative contributions of the two pathways by increasing the attention paid to the nonlexical pathway when it is to their advantage to do so. The circumstance in which it is to their advantage to do so is when most letter strings being presented are either regular words or nonwords. In such circumstances, the expectation is that the frequency effect should decrease for regular word targets and the regularity effect should increase for low-frequency word targets. As discussed, the previous literature has provided some evidence for this type of modulation of the frequency effect but very little evidence for this type of modulation of the regularity effect.

With respect to the modulation in the frequency effect, the point should be made that this account also makes a second prediction. That is, to the extent that the frequency effect is a lexical route effect, there should only be a reduction in the size of the frequency effect for words that can benefit from use of the nonlexical route. Thus, exception words, which do not benefit from the use of the nonlexical route, should not show a reduction in the size of their frequency effect. It is worth keeping in mind that this particular prediction was not examined in any of the papers showing a reduction in the regular word frequency effect. That is, Baluch and Besner (1991) did not evaluate whether there was a reduction in the frequency effect for "opaque" Persian words when nonword fillers were included, Simpson and Kang (1994) did not evaluate whether there was a reduction in the frequency effect for Hanza words when Hangul word fillers were included, and Decker et al. (1999) did not evaluate whether there was a reduction in the frequency effect for exception words when regular word fillers were used.

It is relevant to note, however, that Jared (1997) did manipulate consistency (rather than regularity) and frequency orthogonally. Interestingly, she observed a reduction in the size of the frequency effect for her inconsistent target words in the nonword filler condition (relative to the inconsistent word filler condition), a reduction that was no smaller than the reduction for the consistent words. If these results extend to the manipulation of regularity (rather than consistency), we would have further evidence that the cause of the reduction in the size of the frequency effect was not a shift in the relative contributions of the two pathways.
To this point, we have been discussing the attentional control hypothesis purely in terms of a classic dual-route framework (e.g., Coltheart et al., 1993). As pointed out by Zevin and Balota (2000), the attentional control of pathways hypothesis can also be framed within a PDP system. In this type of system, the two pathways do not represent lexical versus sublexical processing routes but represent a single orthography-to-phonology $(\mathrm{O} \rightarrow \mathrm{P})$ pathway and a pathway from orthography to semantics to phonology (the "semantic" pathway). In general, both of these pathways contribute to the naming process; however, the contribution of the semantic pathway is greater for words with unusual spelling-to-sound mappings (e.g., low-frequency exception words). Within this system, the attentional control hypothesis would simply be that more attention would be allocated to the semantic pathway in a low-frequency exception word environment than in a regular word environment, with the least attention being allocated to the semantic pathway in a nonword environment.

As Zevin and Balota (2000) have discussed, this type of account does not necessarily predict a reduction in the size of the frequency effect as a function of reallocating attention because both the $\mathrm{O} \rightarrow \mathrm{P}$ pathway and the semantic pathway are frequency sensitive. Thus, in order to explain the interaction observed in the present Experiment 2, one would need to add the assumption that the semantic pathway was more frequency sensitive than the $\mathrm{O} \rightarrow$ P pathway. That is, because the exception word environment produced the larger frequency effect and because the exception words should cause participants to allocate more attention to the semantic pathway, one must assume that the semantic pathway is more frequency sensitive than the $\mathrm{O} \rightarrow \mathrm{P}$ pathway.

This assumption, however, immediately causes problems when this framework is applied to the results from Experiment 1. If the semantic pathway really were more frequency sensitive than the $\mathrm{O} \rightarrow \mathrm{P}$ pathway, then the nonword environment in Experiment 1 should have produced the smaller frequency effect. That is, in this condition, there should have been virtually no motivation to allocate attention to the semantic pathway. As a result, the frequency effect should have been much smaller in the nonword environment than in the exception word environment. Contrary to this prediction, however, as noted, there was no hint of modulation in the size of the frequency effect as a function of filler type in Experiment 1.

This framework has similar problems with respect to the regularity effect. To begin with, if one assumes that more attention is being allocated to the semantic pathway in the exception word environment, that environment should produce the most rapid naming of low-frequency exception words. Yet, there was no evidence of such an effect in either Experiment 1 or Experiment 2, although naming of exception words was slightly more accurate in this environment. With respect to the low-frequency regular words, it isn't at all clear whether a shift of attention allocation would affect their naming latencies because it isn't clear which pathway is more effective at processing 
those words. However, in order to explain the filler effect on latency for low-frequency regular words in Experiment 2, one would have to assume that those words are processed more efficiently on the $\mathrm{O} \rightarrow \mathrm{P}$ pathway. If so, the expectation would be that the nonword environment in Experiment 1 should also have produced short latencies for the low-frequency regular words. Again, this expectation was not confirmed in the data.

In conclusion, it doesn't appear that the attentional control of pathways hypothesis can explain the pattern of data reported here regardless of whether that hypothesis is framed in terms of classical dual-route models or in terms of PDP models. We should emphasize that we are not claiming that the present results are problematic for the models themselves: Rather, the results are only problematic for the hypothesis that different pathways for generating phonology from orthography can be strategically turned up or down as a function of filler type. Instead, these results are much better explained in terms of the actions of a time criterion, a concept that can be readily mapped onto either a dual-route or a PDP framework.

In essence, what the time criterion does is to set a point in time at which the participant attempts to respond. The point in time at which the time criterion is set changes as a function of the ease of naming the fillers in a trial block. When the two filler types have fairly similar latencies, changes in the time criterion are minimal, as are changes in target latencies and effect sizes. Only when the two filler types have somewhat different latencies is there a change in the position of the time criterion and, hence, in the target latencies. The present data as well as past results (e.g., Baluch \& Besner, 1991; Decker et al., 1999; Simpson \& Kang, 1994; Zevin \& Balota, 2000) suggest that these changes are smaller for the high-frequency regular words- the targets that are named faster than the faster fillers. As a result, it is much easier to produce a decrease in the regular word frequency effect (an effect that involves high-frequency words) than it is to produce a change in the low-frequency regularity effect (an effect that does not).

\section{REFERENCES}

Batyen, R. H., Piepenbrock, R., \& van Rijn, H. (1993). The CELEX lexical database [CD-ROM]. Philadelphia: University of Pennsylvannia, Linguistic Data Consortium.

BALUCH, B., \& BESNER, D. (1991). Visual word recognition: Evidence for the strategic control of lexical and non-lexical routines in oral reading. Journal of Experimental Psychology: Learning, Memory, \& Cognition, 17, 644-652.

Coltheart, M. (1978). Lexical access in simple reading tasks. In G. Underwood (Ed.), Strategies of information processing (pp. 151-216). New York: Academic Press.

Coltheart, M., Curtis, B., Atkins, P., \& Haller, M. (1993). Models of reading aloud: Dual-route and parallel-distributed processing approaches. Psychological Review, 100, 589-608.

Coltheart, M., \& RASTLE, K. (1994). Serial processing in reading aloud: Evidence for dual-route models of reading. Journal of Experimental Psychology: Human Perception \& Performance, 20, 1197-1211.

Coltheart, M., Rastle, K., Perry, C., Langdon, R., \& Ziegler, J.
(2001). DRC: A dual route cascaded model of visual word recognition and reading aloud. Psychological Review, 108, 204-256.

Decker, G., Simpson, G. B., Yates, M., \& Adamopolous, A. C. (1999, November). Flexible use of lexical and phonological information in word recognition. Poster presented at the annual meeting of the Psychonomic Society, Los Angeles.

JARED, D. (1997). Evidence that strategy effects in word naming reflect changes in output timing rather than changes in processing route. Journal of Experimental psychology: Learning, Memory, \& Cognition, 23, 1424-1438.

JARED, D. (2002). Spelling-sound consistency and regularity effects in word naming. Journal of Memory \& Language, 46, 723-750.

Kinoshita, S., \& LuPKer, S. J. (2001, November). Filler type effects in naming: Control of pathways or time criterion? Paper presented at the annual meeting of the Psychonomic Society, Orlando, FL.

LuPKer, S. J., Brown, P., \& Colombo, L. (1997). Strategic control in a naming task: Changing routes or changing deadlines? Journal of Experimental Psychology: Learning, Memory, \& Cognition, 23, 570-590.

Monsell, S., Patterson, K. E., Graham, A., Hughes, C. H., \& MilRoY, R. (1992). Lexical and sublexical translations of spelling to sound: Strategic anticipation of lexical status. Journal of Experimental Psychology: Learning, Memory, \& Cognition, 18, 452-467.

Raman, I., Baluch, B., \& Besner, D. (2000, July). A critical test of the changing routes versus changing deadlines debate in print-to-sound translation. Paper presented at the joint meeting between the Experimental Psychological Society and the Canadian Society for Brain, Behaviour, and Cognitive Science, University of Cambridge.

Rastle, K., \& Coltheart, M. (1999). Serial and strategic effects in reading aloud. Journal of Experimental Psychology: Human Perception \& Performance, 25, 482-503.

Seidenberg, M. S., Waters, G. S., Barnes, M. A., \& Tanenhaus, M. K. (1984). When does irregular spelling or pronunciation influence word recognition? Journal of Verbal Learning \& Verbal Behavior, 23, 383-404.

Simpson, G. B., \& KANG, H. (1994). The flexible use of phonologicalinformation in word recognition in Korean. Journal of Memory \& Language, 33, 319-331.

TABossi, P., \& LAGHI, L. (1992). Semantic priming in the pronunciation of words in two writing systems: Italian and English. Memory \& Cognition, 20, 303-313.

TAYLOR, T. E., \& LUPKER, S. J. (2001). Sequential effects in naming: A time-criterion account. Journal of Experimental Psychology: Learning, Memory, \& Cognition, 27, 117-138.

Woollams, A. M., \& Kinoshita, S. (1997, April). Strategic modification of response criterion for initiation of articulation in speeded naming. Paper presented at the twenty-fourth Annual Experimental Psychology Conference, Deakin University, Geelong, Victoria, Australia.

Zevin, J. D., \& Balota, D. A. (2000). Priming and attentional control of lexical and sublexical pathways during naming. Journal of Experimental Psychology: Learning, Memory, \& Cognition, 26, 121-135.

\section{NOTES}

1. One notable exception to this pattern has been reported by Zevin and Balota (2000, Experiment 2), who found that the regularity effect was magnified in the nonword filler condition relative to the exception word filler condition. However, using their multiple prime presentation procedure with a different set of target words, we (Kinoshita \& Lupker, 2001) could not replicate their findings: The regularity effect remained constant across the filler conditions. We have argued that the results reported by Zevin and Balota are due to the nature of their target stimuli (their lack of familiarity). We will not discuss the details of this argument here, and for the present purposes, we simply point out that Zevin and Balota's finding of a modulation of the regularity effect has not been replicated.

2 . When a null hypothesis is not rejected, the question of power inevitably arises. In the present experiments, power calculations were done for the frequency by filler type interaction in Experiment 1 and the regularity by filler type interactions in Experiments 1 and 2. The significant 14-msec change in the size of the frequency effect in Experiment 2 was 
used as an estimate of the true effect size for purposes of these calculations. Power calculations show that, if the true sizes of these effects had been $14 \mathrm{msec}$, the power (one-tailed) to detect a significantly larger frequency effect in the exception word filler condition in Experiment 1 would have been .88 and the power to detect a significantly smaller regularity effect in the exception word filler conditions in Experiments 1 and 2 would have been .53 and .35 , respectively.
3. We say the regular word fillers "would" have been named faster than the exception fillers because the filler latencies were not reported by Decker et al. (1999). Similarly, Simpson and Kang (1994, Experiment 4) did not report their filler latencies. However, in both cases, it may be reasonably assumed that the regular word fillers (or Hangul word fillers) were named faster than the exception word fillers (or Hanza word fillers) given what is known about these types of stimuli.

\section{APPENDIX A}

Target Stimuli

\section{High-Frequency Regular Words}

black, ship, claim, case, dance, drink, fact, girl, hair, hand, point, range, serve, street, wife, blue, bring, clay, cattle, club, deep, film, green, help, plane, peace, reach, shape, stop, wish

\section{Low-Frequency Regular Words}

bait, shun, carve, cream, doom, droop, flip, glide, hoarse, helm, plum, roach, surge, sleek, wink, bulb, broth, coach, creep, cage, dump, fuss, goose, huddle, pulp, prune, roast, sheen, sock, wipe

\section{Low-Frequency Exception Words}

beau, chef, chord, cough, deaf, dough, fete, guild, hearse, hood, pear, realm, seize, suite, wolf, bowl, blown, caste, chasm, comb, debt, feud, guise, hearth, pint, plaid, reign, shove, soot, womb

\section{APPENDIX B \\ List of Filler Words}

\section{Low-Frequency Exception Word Fillers (used in both ex- periments)}

aisle, aunt, axe, brooch, bury, choir, chute, couth, crepe, draught, dread, flood, flown, gaol, ghoul, glove, glow, gnome, heir, hoof, leapt, mould, niche, pearl, pique, psalm, rogue, sew, sheik, shoe, sieve, sleight, sown, sponge, spook, steak, suave, suede, swamp, sweat, sword, tomb, tsar, wool, yacht

\section{Nonword Fillers (used in Experiment 1)}

anks, antle, ard, bame, blouch, canks, cound, crope, darge, drestle, enst, fitch, flink, gart, gotch, gream, gurp, hond, jort, leath, morst, norld, pench, plang, raste, seft, sheft, shipe, shud, sim, sking, slaid, sloon, smank, smeed, spant, spooch, spudge, srope, steen, sunch, tard, thore, wike, yeach

\section{Low-Frequency Regular Word Fillers (used in Experiment 2)}

apple, arch, ash, baffle, bean, crust, cling, crate, craze, dwindle, drake, feast, flake, germ, grape, glint, goat, notch, hail, heel, ledge, mulch, nudge, pinch, paste, snail, retch, sip, shine, shin, spill, sniffle, sore, stink, spoon, stain, speck, scarf, swell, swine, swipe, tame, teen, weed, yeast 